\title{
Effect of green tea as a protective measure against dental erosion in coronary dentine
}

\section{Maria Denise Rodrigues DE MORAES(a) \\ Jéssica Rodrigues Mendes CARNEIRO(b) \\ Vanara Florêncio PASSOS(a) Sérgio Lima SANTIAGO ${ }^{(b)}$}

(a) Universidade de Fortaleza - Unifor, Dentistry Course, Fortaleza, CE, Brazil.

(b) Universidade Federal do Ceará - UFC, Faculty of Pharmacy, Dentistry and Nursing, Fortaleza, CE, Brazil.

Declaration of Interests: The authors certify that they have no commercial or associative interest that represents a conflict of interest in connection with the manuscript.

Corresponding Author:

Sérgio Lima Santiago

E-mail: sergiosantiago@yahoo.com

DOI: 10.1590/1807-3107BOR-2016.vol30.0013

Submitted: Mar 25, 2015

Accepted for publication: Jun 30, 2015

Last revision: Aug 31, 2015

\begin{abstract}
The aim of this study was to evaluate the effect of green tea as a protective measure on eroded dentin. Disks of human coronary dentin were selected based on surface hardness and randomly assigned to 3 groups $(n=10)$ : DW - distilled water, $\mathrm{CHX}-0.2 \%$ chlorhexidine digluconate, and GT - green tea. The disks were allowed to acquire pellicle for 2 hours and were then subjected to 3 cycles per day of demineralization $\left(\mathrm{C}_{6} \mathrm{H}_{8} \mathrm{O}_{7} 0.05 \mathrm{M}, \mathrm{pH} 3.75,60 \mathrm{~s}\right)$, treatment (DW or CHX or GT, $5 \mathrm{~min}$ ) and remineralization (artificial saliva, $60 \mathrm{~min}$ ) over a period of 3 days. Changes in the dentin were determined by loss of surface hardness (\%SHL) and mechanical profilometry analysis at the end of each day. Data were analyzed by two-way ANOVA followed by Tukey's test for \%SHL and profilometry $(\mathrm{p}<0.05)$. Significant reductions in dentin hardness loss were observed only for the CHX group when compared to the DW group $(\mathrm{p}<0.05)$. However, there was no significant difference between the CHX and GT groups ( $p>0.05)$. A significant difference was observed between DW and GT treatments for wear and roughness measurements $(p<0.05)$. The green tea extract solution was able to reduce the wear and roughness caused by dentin erosion under the conditions of this study.
\end{abstract}

Keywords: Tooth Erosion; Dentin; Hardness; Matrix Metalloproteinase Inhibitors; Camellia sinensis.

\section{Introduction}

Erosive demineralization of the tooth crown is characterized by initial softening of the enamel surface. This process is followed by continuous layer-by-layer dissolution of the enamel crystals, leading to a permanent loss of tooth volume with a softened layer at the surface. The dentin becomes increasingly exposed in advanced stages and exposes the organic matrix to breakdown by host-derived enzymes, such as matrix metalloproteinases (MMPs) present in dentin and saliva. ${ }^{1,2,3}$ MMPs act in the chemical degradation of the organic matrix of dentin and play an important role in the progression of dentin erosion. ${ }^{4,5,6}$

Protective measures to reduce dental erosion, such as laser therapy and topical fluoride, have been investigated. Most of these treatments are based on the action of fluoride, which is available in dentifrices, solutions, or varnishes, although the role of fluoride in the protection of dental erosion is still controversial. ${ }^{7,8,10}$ 
In recent years, some in situ studies have shown that commercial green tea and a rinse containing green tea extract were able to reduce erosive and erosive/abrasive dentin wear. ${ }^{11,12}$ These products are rich in polyphenols and have been reported to be inhibitors of the activity of different metalloproteinases. ${ }^{11,13}$ It has been suggested that the use of synthetic inhibitors of MMPs could be used to control the loss of the dentin matrix. ${ }^{5,14}$ However, MMPs are secreted as inactive precursors (pro-forms), requiring activation by a low $\mathrm{pH}$ to degrade extracellular matrix components. ${ }^{1}$

Therefore, the demineralization process is increased by the action of acids on the organic matrix of dentin and the activation of the MMPs. ${ }^{12}$ To minimize this process, green tea has a significant inhibitory effect on MMPs and dentin demineralization. ${ }^{15,16,17}$ The reduced daily exposure to acid may be minimized by the use of metalloproteinase inhibitors, which protect the organic matrix as a barrier to ion diffusion.

Other studies have attempted to evaluate the protective effect of green tea against wear promoted by erosion; however, there are still doubts concerning the maintenance of inhibitory action in the MMP after successive cyclical acidic exposures. It might be interesting to investigate the effectiveness and substantivity of these substances on the surface of the eroded dentin in cycles of erosive challenge. The absence of studies that simulate the erosion of dentin through short cyclic acid challenges, both daily and intermittent, reinforces the need for further studies.

Thus, the objective of this in vitro study was to assess the protective effect of green tea on dentin demineralization in three days of cyclic erosion by assessing the percentage of superficial hardness loss (\%SHL), roughness and wear.

\section{Methodology}

\section{Experimental design}

This in vitro study was approved by the local research and ethics committee (protocol \# 175/2010). This is a blinded experimental study design with three groups $(\mathrm{n}=10)$, with two factors: time (one, two and three days of the experimental cycle) and treatments (distilled water (DW; G1- control), 0.2\% chlorhexidine (CHX; G2) and green tea (GT; G3;
EGCG concentration $0.0014 \%$ ). The treatments were applied by immersion under agitation for five minutes, and the specimens were randomly assigned to the defined treatments. The dependent variables were the percentage of surface hardness loss (\%SHL), as quantitatively evaluated by differences in the mean values of superficial microhardness after treatment, and dentinal wear and roughness, as assessed by the contact profilometer Hommel Tester T1000 (Jenoptik, Schwenningen, Germany).

\section{Specimen preparation}

Coronary dentin specimens were prepared from human third molars that had been stored in $0.01 \%(\mathrm{w} / \mathrm{v})$ thymol solution at $4^{\circ} \mathrm{C}$. Dentin disks were obtained using a longitudinal coupled double-sided diamond disk in a IsoMet slow speed saw (Buehler, Lake Bluff, USA). The dimensions of each disk depended on the diameter of the tooth. Sequentially, the specimens were ground in a water-cooled mechanical grinder (Arotec S.A., Cotia, Brazil) using 400-, 600-, 800- and 1200-grit aluminum oxide abrasives and polished with felt paper and $1 \mu \mathrm{m}$ diamond spray (Extec Corp., Enfield, USA). The surface hardness values were determined using a Knoop diamond FM100 (Future-Tech Corp., Kawasaki, Japan), making five indentations with a load of $10 \mathrm{~g} / 5 \mathrm{~s}, 100 \mu \mathrm{m}$ apart from each other, at the center of the specimens. Thirty dentin disks presenting a mean hardness of $55.81 \pm 6.20$ Knoop hardness number $(\mathrm{KHN})$ were selected and randomly assigned using a computer-generated randomization list into three experimental groups (Microsoft Excel 2007).

Nail varnish was applied on half of the surface of each specimen to serve as the reference area for profilometry analysis. The exposed area was subjected to the acid challenge.

\section{Pellicle formation}

On each experimental day, five volunteers without erosion, salivary dysfunction or active carious lesions donated fresh saliva samples. The secretion of saliva was stimulated by chewing on paraffin wax for five minutes. Saliva from the first minute of chewing was swallowed, and the rest was collected and deposited into a $50 \mathrm{ml}$ centrifuge tubule. The saliva samples were centrifuged for $10 \mathrm{~min}$ at $2000 \mathrm{rpm}$ in 
a pre-cooled centrifuge $\left(4^{\circ} \mathrm{C}\right)$ NT-815 (Novatecnica, Piracicaba, Brazil). The clear fluid above the sediments was pooled and used for pellicle formation..$^{18}$ Prior to erosive challenge, each group of dentin disks was independently immersed in clarified saliva and incubated under agitation at $100 \mathrm{rpm}$ in an oscillating table (TE143, Tecnal, Piracicaba, Brazil) at $37^{\circ} \mathrm{C}$ (Olidef $\mathrm{CZ}$, Ribeirão Preto, Brazil) for two hours before each experimental day to simulate the environment of the oral cavity.

\section{Experimental procedure}

The study used cyclic procedures repeated over a three-day period, including pellicle formation, erosion, treatments with test solutions (DW, CHX, GT) and remineralization with freshly prepared artificial saliva (1.5 mM Ca; $0.9 \mathrm{mM}$ PO4; $150 \mathrm{mM}$ $\mathrm{KCl}$ and $0.1 \mathrm{M}$ Tris buffer, $\mathrm{pH}$ 7.0), considering the influence of variables such as stirring, temperature and exposure time (Figure). ${ }^{19}$ The $0.2 \%$ chlorhexidine digluconate solution was provided by a pharmacy, and the green tea (Dr Oetker, São Paulo, Brazil) was freshly prepared according to the manufacturer's instructions at the beginning of each experimental day. The concentration of epigallocatechin gallate (EGCG) in this green tea was $0.0014 \%$, as assessed using a spectrophotometer, and the $\mathrm{pH}$ was 5.45 .

\section{Erosion cycling model}

After pellicle formation, each disk was submitted to a citric acid solution for 60 seconds. The acid

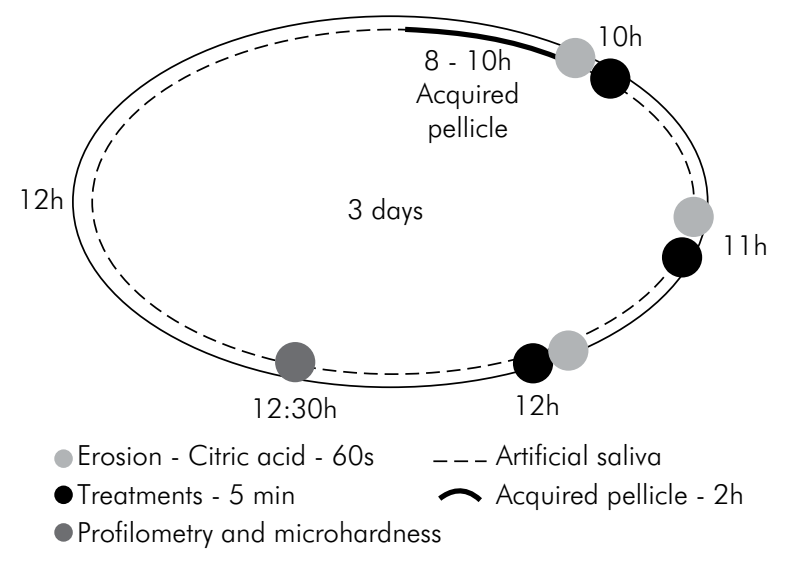

Figure. Erosion cycle model and treatments. challenge was performed using $0.05 \mathrm{M}$ dehydrated citric acid, pH 3.75 (Dinâmica ${ }^{\circledR}$, Diadema, Brazil). Each disk was then rinsed with distilled water and treated for 5 minutes with the treatment solution (DW, CHX, GT) and then immersed in artificial saliva for 1 hour, performed under agitation at $100 \mathrm{rpm}$ and $37^{\circ} \mathrm{C}$ (Figure).

This cycle was repeated three times a day for three days. At the end of each experimental day, the hardness, wear and surface roughness analysis of each specimen were measured. ${ }^{20}$

\section{Percentage of surface hardness loss assessment}

Immediately after each experimental day, the slabs were placed in the hardness machine, and five new indentations were made using a Knoop diamond under a $10 \mathrm{~g}$ load for five seconds (SHafter), with the indentations spaced $100 \mu \mathrm{m}$ apart from the previous measurements (SHbefore). The percentage of SH loss (\%SHL) was then calculated for each day, according to the following equation: \%SHL $=[($ SHbefore - SHafter $)$ x100/ SHbefore].

\section{Measurement of dentin surface loss}

Measurements of dentin surface loss were performed using the stylus profilometer, after each experimental day. The difference between the heights of the surfaces of the reference and the treated areas was evaluated. Before analysis, the nail varnish was carefully removed, exposing the untreated reference areas. On each sample, at intervals of $100 \mu \mathrm{m}$, five profile traces $(1.5 \mathrm{~mm}$ in length) were recorded, and the levels of dentin wear were determined in relation to the reference surfaces. For each sample, the mean values obtained from the five traces were calculated. ${ }^{21}$

\section{Measurement of surface roughness}

Surface roughness was described by the arithmetic mean of the absolute ordinate values Ra (average roughness as per ISO 4287) of 5 measurements made in each disk. ${ }^{22,23}$ In profilometry, the surface of a specimen was scanned using a stylus with a diamond to generate a two- or three-dimensional profile using a contact measuring device. ${ }^{24}$ 


\section{Statistical analysis}

Statistical procedures were performed with the Statistical Package for Social Sciences (SPSS 17.0 for Windows, SPSS Inc., Chicago, USA). A Kolmogorov-Smirnoff test was applied to all groups to test for the normal distribution of errors. Because the values were normally distributed across all groups, two-way ANOVA and Tukey's post hoc tests were used for comparative purposes. The level of significance was set at $5 \%$.

\section{Results}

Table presents the means of dentin hardness loss, wear and roughness values found for all the treatments evaluated in the three-day experiment.

In relation to dentin hardness loss, dental wear and roughness, two-way ANOVA revealed a significant difference among the treatments tested $[(\mathrm{p}<0.001 ; \mathrm{F}=3.3),(\mathrm{p}<0.001 ; \mathrm{F}=9.7)$, and $(\mathrm{p}<0.001$; $\mathrm{F}=8.3$ ), respectively], as well as the duration of demineralization represented by the number of experimental days $[(\mathrm{p}<0.001 ; \mathrm{F}=69.7),(\mathrm{p}<0.001$; $\mathrm{F}=11.4)$, and $(\mathrm{p}<0.001 ; \mathrm{F}=49.0)$, respectively]. Furthermore, the interaction between the factors was significant for the loss of dentin hardness ( $p=0.009$; $F=3.6)$. However, the interaction between the factors was not significant for dental wear $(\mathrm{p}=0.745 ; \mathrm{F}=0.4)$ and for roughness $(p=0.782 ; F=0.4)$. Significant differences in dentin hardness loss were observed only for the CHX group when compared to the DW group ( $\mathrm{p}<0.05)$. However, there was no significant difference between CHX and GT ( $p>0.05)$.

For dentin hardness loss and dental wear, it was observed that with an increase in the number of experimental days, all specimens displayed

Table. Means and standard deviations of the loss of dentin hardness, wear and roughness values for all the treatments evaluated.

\begin{tabular}{lccc}
\hline \multirow{2}{*}{ Group } & \multicolumn{3}{c}{ Analyses } \\
\cline { 2 - 4 } & Dentin Hardness Loss & Wear $(\mu \mathrm{m})$ & Roughness \\
\hline DW & $55.67(9.13) \mathrm{a}$ & $2.07(0.77) \mathrm{a}$ & $0.25(0.04) \mathrm{a}$ \\
CHX & $61.83(7.59) \mathrm{b}$ & $1.78(0.78) \mathrm{ab}$ & $0.22(0.03) \mathrm{ab}$ \\
GT & $58.78(9.48) \mathrm{ab}$ & $1.25(0.48) \mathrm{b}$ & $0.20(0.04) \mathrm{b}$ \\
\hline
\end{tabular}

Different lowercase letters indicating significant differences between groups in columns. statistically significant surface softening from day 1 to day 2 , which stabilized at day 3 , showing that citric acid was effective in eroding the dentin surface. The green tea was more effective than DW in protecting the human dentin against wear caused by the erosion of citric acid $(p<0.001)$. Additionally, there was no significant difference between CHX and GT $(p<0.05)$.

The chlorhexidine behaved similarly to the control $(p=0.053)$ in protecting the human dentin against surface roughness caused by the erosion of citric acid. On the other hand, the green tea was more effective than the control $(\mathrm{p}<0.001)$.

\section{Discussion}

The present in vitro de-remineralization cycling model investigated green tea with respect to its capacity to protect human dentin from erosion. This study confirmed the expected surface softening and dentin tissue loss due to the action of citric acid, even after pellicle formation for two hours before each experimental day. The use of an in vitro multiple-exposure acid model allowed for a better understanding of the erosive challenges faced by the dentition while performing a controlled investigation and reducing the experimental time and cost. ${ }^{25}$

The results of this study disagree with the results of Mirkarimi and Toomarian ${ }^{26}$ because an increase in surface hardness values after immersion in green tea solution was not observed in the current study. However, the erosive challenge used in their study was shorter and lower than that used in the present study. The dentin disks subjected to this erosive cycle presented higher percentages of change in hardness, which may have influenced the indentations of the diamond when measuring hardness. Furthermore, the hardness analysis is a sensitive method for detecting changes in the mineral density of artificial eroded/abraded lesions in an enamel substrate but not in dentin; ${ }^{27}$ therefore, another analysis, profilometry, was added to the current study.

Under great acid challenge, profilometric analysis is widely used to measure the loss of enamel and dentin surfaces. ${ }^{27,28}$ The results of the present study corroborate those of Magalhães et al. ${ }^{12}$ and Kato et al. ${ }^{11}$ because the green tea was able to reduce dentin wear in the current study. A possible mechanism of 
action for the reduction in dentin loss might be the inhibition of MMPs by chlorhexidine and green tea extract solution. ${ }^{12}$ Another study that involved cyclic acid challenge also used profilometry to measure the wear of dentin; Barbosa et al. ${ }^{29}$ concluded that the supplementation of soft drinks with green tea extract might be a viable alternative to reduce the erosive potential against dentin.

In this study, a cycling model was used, employing citric acid three times a day over a period of 3 days. On the $3^{\text {rd }}$ day of the erosive challenge, the surface roughness and dentin wear were worse than in the first day, showing that the acid used in this study was effective in eroding the dentin surface. It is also probable that the low $\mathrm{pH}$ of the acid induced the activation of dentin-derived MMPs. ${ }^{11}$

The $1 \mu \mathrm{m}$ of wear protection observed in this in vitro study correlates with the erosive cycle, which involved short periods of acid exposure and remineralization. Magalhães et al. ${ }^{12}$ performed a more aggressive challenge (4 times/day, Coca Cola, $5 \mathrm{~min}$ ) than in this study (3 times/day, citric acid, $1 \mathrm{~min}$ ) and observed a similar outcome. Additionally, in that study, green tea extract with approximately $30 \pm 3 \%$ of catechin was used, while the present study used a commercial product, which limited the concentration of catechin $(0.0014 \%)$.

One possible mechanism of action of green tea on the reduction of dentin erosion could be the inhibition of MMPs. If it is true, the component responsible for this effect may be polyphenols. Green tea polyphenols, especially epigallocatechin-3-gallate (EGCG), were

\section{References}

1. Buzalaf MA, Kato MT, Hannas AR. The role of matrix metalloproteinases in dental erosion. Adv Dent Res. 2012;24(2):72-6. doi:10.1177/0022034512455029

2. Pashley DH, Tay FR, Yiu C, Hashimoto M, Breschi L, Carvalho RM et al. ITO S. Collagen degradation by host-derived enzymes during aging. J Dent Res. 2004;83(3):216-21. doi:10.1177/154405910408300306

3. Hannas AR, Pereira JC, Granjeiro JM, Tjäderhane L. The role of matrix metalloproteinases in the oral environment. Acta Odontol Scand. 2007;65(1):1-13. doi:10.1080/00016350600963640

4. Hara AT, Ando M, Cury JA, Serra MC, Gonzalez-Cabezas C, Zero DT. Influence of the organic matrix on root found to have potent and distinct inhibitory activity against MMPs in cell culture tests. ${ }^{11}$ The green tea polyphenols and their major constituent, EGCG, have been shown to inhibit metalloproteinases in other areas of medicine and dentistry. ${ }^{30,31}$ It can be speculated that the small concentration of EGCG in the tea used in the present study can justify the unsatisfactory results in protecting the loss of dentin hardness. However, it must be acknowledged that the protocol employed in the present study does not suggest that the effect of green tea on the reduction of the wear and roughness of dentin specimens is due to its inhibitory action on MMP activity, as we did not test this directly.

Because the erosion/remineralization cycle was performed under in vitro conditions, it is clear that the results are not completely transferable to an in vivo situations, since the natural protective effects of the oral cavity are lacking. However, the present study utilized variables such as the erosive solution, stirring method and temperature in order to replicate the oral environment. Thus, the use of green tea might be a viable alternative as a protective measure against erosive wear in short acid exposures. However, further studies are necessary to explore the inhibitory action of green tea solution on MMPs present in erosively demineralized dentin.

\section{Conclusion}

According to the conditions of the present study, the use of green tea extract is a promising protective measure in reducing dentin erosion, as it has a protective effect on the dentin roughness and wear caused by erosion.

dentine erosion by citric acid. Caries Res. 2005;39(2):134-8. doi:10.1159/000083159

5. Kato MT, Leite AL, Hannas AR, Calabria MP, Magalhães AC, Pereira JC et al. Impact of protease inhibitors on dentin matrix degradation by collagenase. J Dent Res. 2012;91(12):1119-23. doi:10.1177/0022034512455801

6. Buzalaf MA, Hannas AR, Kato MT. Saliva and dental erosion. J Appl Oral Sci. 2012;20(5):493-502. doi:10.1590/S1678-77572012000500001

7. Messias DC, Maeda FA, Turssi CP, Serra MC. Effect of dentifrices against hydrochloric acid-induced erosion. Oral Health Prev Dent. 2011;9(3):269-73. 
8. Passos VF, Melo MA, Silva FF, Rodrigues LK, Santiago SL. Effects of diode laser therapy and stannous fluoride on dentin resistance under different erosive acid attacks. Photomed Laser Surg. 2014;32(3):146-51. doi:10.1089/pho.2013.3629

9. Passos VF, Santiago SL, Tenuta LM, Cury JA. Protective effect of $\mathrm{NaF} /$ triclosan/copolymer and MFP dentifrice on enamel erosion. Am J Dent. 2010;23(4):193-5.

10. Wiegand A, Attin T. Influence of fluoride on the prevention of erosive lesions: a review. Oral Health Prev Dent. 2003;1(4):245-53.

11. Kato MT, Magalhaes AC, Rios D, Hannas AR, Attin T, Buzalaf MA. Protective effect of green tea on dentin erosion and abrasion. J Appl Oral Sci. 2009;17(6):560-4. doi:10.1590/S1678-77572009000600004

12. Magalhães AC, Wiegand A, Rios D, Hannas A, Attin T, Buzalaf MA. Chlorhexidine and green tea extract reduce dentin erosion and abrasion in situ. J Dent. 2009;37(12):994-8. doi:10.1016/j.jdent.2009.08.007

13. Sulkala M, Larmas M, Sorsa T, Salo T, Tjäderhane L. The localization of matrix metalloproteinase-20 (mmp-20) in mature human teeth. J Dent Res. 2002;81(9):603-7. doi:10.1177/154405910208100905

14. Gendron R, Grenier D, Sorsa T, Mayrand D. Inhibition of the activities of matrix metalloproteinases 2, 8, and 9 by chlorhexidine. Clin Diagn Lab Immunol. 1999;6(3):437-9.

15. Demeule M, Brossard M, Pagé M, Gingras D, Béliveau R. Matrix metalloproteinase inhibition by green tea catechins. Biochim Biophys Acta. 2000;1478(1):51-60. doi:10.1016/S0167-4838(00)00009-1

16. Kato MT, Leite AL, Hannas AR, Buzalaf MA. Gels Containing MMP Inhibitors Prevent Dental Erosion in situ. J Dent Res. 2010;89(5):468-72. doi:10.1177/0022034510363248

17. Silveira C, Oliveira F, Santos ML, Freitas T, Imparato JC, Magalhães AC. Anarcadic acid from brazilian cashew nut trees reduces dentine erosion. Caries Res. 2014;48(6):549-56. doi:10.1159/000358400

18. Melo MA, Passos VF, Alves JJ, Barros EB, Santiago SL, Rodrigues LK. The effect of diode laser irradiation on dentin as a preventive measure against dental erosion: an in vitro study. Lasers Med Sci. 2011;26(5):615-21. doi:10.1007/s10103-010-0865-y

19. Young A, Tenuta LM. Initial erosion models. Caries Res. 2011;45 Suppl 1:33-2. doi:10.1159/000325943

20. Caneppele TM, Jeronymo RD, Di Nicoló R, Araújo MA, Soares LE. In vitro assessment of dentin erosion after immersion in acidic beverages: surface profile analysis and energy-dispersive x-ray fluorescence spectrometry study. Braz Dent J. 2012;23(4):373-8. doi:10.1590/S0103-64402012000400011

21. Moron BM, Miyazaki SS, Ito N, Wiegand A, Vilhena F, Buzalaf MA et al. Impact of different fluoride concentrations and ph of dentifrices on tooth erosion/abrasion in vitro. Aust Dent J. 2013;58(1):106-11. doi:10.1111/adj.12016

22. Jung M, Eichelberger K, Klimek J. Surface geometry of four nanofiller and one hybrid composite after one-step and multiple-step polishing. Oper Dent. 2007;32(4):347-55. doi:10.2341/06-101

23. Jung M, Sehr K, Klimek J. Surface texture of four nanofilled and one hybrid composite after finishing. Oper Dent. 2007;32(1):45-52. doi:10.2341/06-9

24. Schlueter N, Hara A, Shellis RP, Gans C. Methods for the measurement and characterization of erosion in enamel and dentine. Caries Res. 2011;45Suppl 1:13-23. doi:10.1159/000326819

25. Shellis RP, Ganss C, Ren Y, Zero DT, Lussi A. Methodology and models in erosion research: discussion and conclusions. Caries Res. 2011; 45 Suppl 1:69-77. doi:10.1159/000325971

26. Mirkarimi M, Toomariam L. Effect of green tea extract on the treatment of dentin erosion: an in vitro study. J Dent (Tehran). 2012;9(4):224-8.

27. Passos VF, Melo MA, Vasconcellos AA, Rodrigues LK, Santiago SL. Comparison of methods for quantifying dental wear caused by erosion and abrasion. Microsc Res Tech. 2013;76(2):178-83. doi:10.1002/jemt.22150

28. Ganss C, Lussi A, Scharmann I, Weigelt T, Hardt M, Klimek $\mathrm{J}$ et al. Comparison of calcium analysis, longitudinal microradiography and profilometry for the quantitative assessment of erosion in dentine. Caries Res. 2009;43(6):422-9. doi:10.1159/000252975

29. Barbosa CS, Kato MT, Buzalaf MA. Effect of supplementation of soft drinks with green tea extract on their erosive potential against dentine. Aust Dent J. 2011;56(3):317-21. doi:10.1111/j.1834-7819.2011.01338.x

30. Deb G, Thakur V, Limaye A, Gupta S. Epigenetic induction of tissue inhibitor of matrix metaloproteinase- 3 by green tea polyphenols in breast cancer cells. Mol Carcinog. 2015;54(6):485-99. doi:10.1002/mc.22121

31. Wen WC, Kuo PJ, Chiang CY, Fu E. Epigallocatechin3 -gallate attenuates porphyromonas gingivalis lipopolysaccharide-enhanced matrix metalloproteinase-1 production through an inhibition of interleukin-6 in gingival fibroblasts. J Periodontol. 2014;85(6):868-75. doi:10.1902/jop.2013.120714 